\title{
Prevalência de Transtornos Mentais Comuns em trabalhadores de restaurantes
}

\author{
Prevalence of Common Mental Disorders in restaurant workers \\ Prevalencia de Trastornos Mentales Comunes en los trabajadores de restaurantes
}

Recebido: 22/02/2021 | Revisado: 03/03/2021 | Aceito: 05/03/2021 | Publicado: 14/03/2021

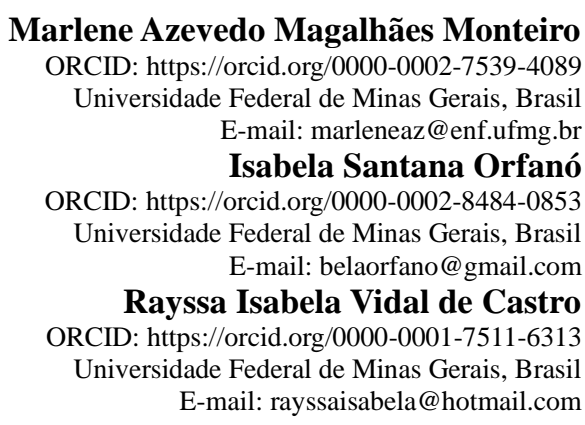

\begin{abstract}
Resumo
Introdução: Os aspectos psicossociais do trabalho são fontes de estresse ocupacional, com impactos na saúde mental. Há poucos estudos relacionando Transtornos Mentais Comuns (TMC) em trabalhadores de restaurantes. Objetivo: analisar a presença e os fatores associados aos Transtornos Mentais Comuns (TMC) em trabalhadores de restaurantes de Belo Horizonte, MG. Metodologia: Foi realizado um estudo transversal, descritivo, com amostra de 87 indivíduos que representou $72,5 \%$ dos trabalhadores distribuídos em quatro restaurantes universitários. Resultados: Entre os trabalhadores dos restaurantes entrevistados, 10,3\% ( $\mathrm{n}=9$ ) apresentaram quadro atual de Transtornos Mentais Comuns (TMC). Foi encontrada associação significativa entre presença de Transtornos Mentais Comuns (TMC) com gênero, acompanhamento psicológico e psiquiátrico, e sem correlação significativa com as demais variáveis estudadas (obesidade, função, jornada de trabalho). Conclusão: Os resultados deste estudo indicam que a prevalência de Transtornos Mentais Comuns (TMC) entre os trabalhadores de restaurantes é uma realidade e que necessita de intervenção para promover melhor qualidade de vida a esses indivíduos.
\end{abstract}

Palavras-chave: Restaurantes; Transtornos mentais comuns; Saúde do trabalhador; Condições de trabalho.

\begin{abstract}
Introduction: Psychosocial aspects of work are sources of occupational stress, with impacts on mental health. There are few studies relating Common Mental Disorders (CMD) in restaurant workers. Objective: to analyze the presence and factors associated with Common Mental Disorders (CMD) in restaurant workers in Belo Horizonte, MG. Methodology: A cross-sectional, descriptive study was carried out, with a sample of 87 individuals who represented 72.5\% of the workers distributed in four university restaurants. Results: Among the restaurant workers interviewed, 10.3\% ( $\mathrm{n}=9)$ presented current Common Mental Disorders (CMD). A significant correlation was found between the presence of Common Mental Disorders (CMD), with gender, psychological and psychiatric monitoring, and with no significant correlation with the other variables studied (obesity, function, working hours). Conclusion: The results of this study indicate that the prevalence of Common Mental Disorders (CMD) among restaurant workers is a reality and that it requires intervention to promote a better quality of life for these individuals.
\end{abstract}

Keywords: Restaurants; Common mental disorders; Occupational health; Working conditions.

\section{Resumen}

Introducción: Los aspectos psicosociales del trabajo son fuentes de estrés laboral, con impactos en la salud mental. Hay pocos estudios relacionados con los trastornos mentales comunes (CMD) en trabajadores de restaurantes. Objetivo: analizar la presencia y factores asociados a los Trastornos Mentales Comunes (DMC) en trabajadores de restaurantes de Belo Horizonte, MG. Metodología: se realizó un estudio descriptivo, transversal, con una muestra de 87 individuos que representó el 72,5\% de los trabajadores distribuidos en cuatro restaurantes universitarios. Resultados: Entre los trabajadores de restaurantes entrevistados, el 10,3\% $(\mathrm{n}=9)$ presentaba Trastornos Mentales Comunes (DMC) actual. Se encontró asociación entre la presencia de Trastornos Mentales Comunes (DMC) con el seguimiento psicológico y psiquiátrico, y sin correlación significativa con el resto de variables estudiadas (obesidad, función, jornada laboral). Conclusión: Los resultados de este estudio indican que la prevalencia de Trastornos Mentales Comunes (CMD) entre los trabajadores de restaurantes es una realidad y que requiere intervención para promover una mejor calidad de vida de estos individuos. 
Palabras clave: Restaurantes; Trastornos mentales comunes; Salud del trabajador; Condiciones de trabajo.

\section{Introdução}

O processo de trabalho é composto por um conjunto de atividades complexo e pelas condições laborais que precisam ser monitoradas constantemente para proporcionar qualidade de vida e bem-estar psicológico aos trabalhadores (Silva \& Borges, 2015; Konzen et al., 2020).

Desta forma, a saúde do trabalhador pode estar comprometida devido a suas interações com as condições de trabalho que estão relacionadas a satisfação na realização das atividades, segurança no trabalho e autonomia em relação à tarefa executada, além das relações estabelecidas dos trabalhadores entre si. Assim, a divisão, a inflexibilidade e o conteúdo das tarefas, surgem como fatores contribuintes do sofrimento mental do trabalhador, podendo resultar em distúrbios psíquicos (Lindstrom, 2005; Härma, Kompier \& Vahiera, 2006; Ganster \& Rosen, 2013).

A saúde mental é um estado de bem-estar no qual um indivíduo percebe suas próprias habilidades, pode lidar com as tensões normais da vida, trabalhar de forma produtiva e é capaz de dar uma contribuição para sua comunidade. A saúde mental é fundamental para a habilidade coletiva e individual de todo ser humano (World Health Organization, 2020).

O Transtorno Mental Comum (TMC) é uma condição de saúde que engloba sintomas psicogênicos compatíveis com depressão ou ansiedade não diagnosticadas. É uma expressão criada para designar sintomas tais como insônia, fadiga, irritabilidade, esquecimento, dificuldade de concentração e queixas somáticas, que demonstram ruptura do funcionamento normal do indivíduo. Além disso, trazem incapacidade funcional e sofrimento para as pessoas que a desenvolvem (Golderg \& Huxley, 1992; Tenório et al., 2016, Sousa et al., 2017).

Estudos mostram uma prevalência de TMC entre $7 \%$ e 30\%, no mundo, e também revelam que cerca de 1/3 dos dias perdidos de trabalho, taxas de mortalidade mais elevadas e prejuízos nas funções sociais e físicas das pessoas estão relacionados a existência de transtornos mentais (Lopes, Faerstein \& Chor, 2003). Além disto, um Relatório da Organização Mundial de Saúde (OMS) aponta que o número de casos de depressão aumentou 18\% entre 2005 e 2015: são 322 milhões de pessoas em todo o mundo, a maioria mulheres (WHO, 2017). No Brasil a depressão atinge 10\% da população (Santana et al., 2016).

O restaurante ou Unidade de Alimentação e Nutrição (UAN) possui o objetivo de administrar e fornecer refeições equilibradas nutricionalmente e com padrão higiênico sanitário (Abreu, Spinelli \& Pinto, 2019). Para o funcionamento adequado de uma UAN, os processos de produção e distribuição das refeições devem acompanhar o porte (quantidade e tipo de refeição ofertada), sendo o número de funcionários, suas habilidades de trabalho e infraestrutura da unidade fatores essenciais (Colares \& Freitas, 2007; Estevam \& Guimarães, 2013).

De acordo com Lourenço e Menezes (2008), o trabalho em uma UAN é caracterizado por manipulação manual intensa durante o processo de pré-preparo e preparo dos alimentos. A realização dessas tarefas vem acompanhada de movimentos repetitivos de membros superiores e coluna, levantamento de pesos excessivos e permanência na postura em pé por períodos prolongados, produção intensa, manipulação de peso excessivo de forma inadequada, além de exposição a condições de risco. Devido ao curto e limitado tempo entre preparo e distribuição das refeições é observado que os manipuladores de alimentos sofrem grande pressão, resultando em um ritmo de trabalho intenso (Abreu, Spinelli \& Pinto, 2019). Diante desse contexto, a saúde do trabalhador é um motivo de preocupação, uma vez que é afetada tanto quanto a sua produtividade (Lindstrom, 2005; Abreu, Spinelli \& Pinto, 2019).

As causas de incidência desses transtornos mentais em trabalhadores de restaurantes são diversas, como as condições físicas de trabalho (Dourado \& Lima, 2011), instalações inadequadas, equipamentos faltantes, ruídos excessivos, altas temperaturas e baixa iluminação, além do esforço intenso, jornada de trabalho prolongada, sobrecarga de tarefas, excessiva 
pressão em função de tempo e prazos. E, ainda, a sua presença nestes trabalhadores pode ser uma resposta ao estresse (Barreto \& Branco, 2000; Bárbaro et al., 2009; Silva, Bernardo \& Souza, 2016), e estão entre as principais causas de absenteísmo e licença médica (Pinto \& Silva, 2016; Niedhammer, Malard \& Chastang, 2015).

Diante do exposto, o objetivo desse estudo foi analisar a presença e os fatores associados aos Transtornos Mentais Comuns (TMC) em trabalhadores de restaurantes de Belo Horizonte, MG.

\section{Metodologia}

Trata-se de um estudo transversal, descritivo, quantitativo e exploratório (Pereira et al., 2018) cuja coleta de dados foi realizada por meio de censo com os trabalhadores de quatro restaurantes universitários do município de Belo Horizonte - MG, no período de março a outubro de 2017. Participaram do estudo 87 indivíduos (72,5\% da amostra), sendo excluídos os contadores e caixas dos restaurantes. Todos os voluntários assinaram o Termo de Consentimento Livre e Esclarecido.

Para a coleta de dados foi utilizado um questionário estruturado em blocos com questões fechadas, aplicado por estudantes capacitados por meio de entrevistas individuais em local e horário de trabalho (Lima et al., 2016). O questionário abrangeu aspectos sociodemográficos (idade, sexo, escolaridade, estado conjugal, renda, número de filhos e de moradores da casa), autoavaliação de saúde, dados antropométricos (altura, peso, IMC e circunferência abdominal) e informações ocupacionais (função, tempo de trabalho e jornada média diária). Para rastrear casos suspeitos de Transtornos Mentais Comuns (TMC) foi utilizado o Self-Reporting Questionnaire (SRQ-20) adaptado (Lima et al., 2016).

Visando atingir ao objetivo proposto foram consideradas as seguintes informações: sexo, idade, função, depressão, transtorno de ansiedade, uso de antidepressivo e/ou calmantes, e algum tipo de acompanhamento psiquiátrico e psicológico, e correlacionando a autoavaliação de saúde com as informações ocupacionais.

O índice de Massa Corporal (IMC) foi calculado a partir dos dados de peso e altura. Este índice é classificado para adultos em: "eutrofia" (valores entre 18,5 kg/m² e 24,9 kg/m²), "sobrepeso" (valores entre $25 \mathrm{~kg} / \mathrm{m}^{2}$ e 29,9 kg/m²), e "obeso" (valores acima de $30 \mathrm{~kg} / \mathrm{m}^{2}$ ) (WHO, 2000). A classificação para circunferência da cintura (CC) foi feita de acordo com a OMS, na qual valores acima de $80 \mathrm{~cm}$ para mulheres, e $94 \mathrm{~cm}$ para homens são considerados "risco elevado para Doenças Cardiovasculares (DCV)". O referencial utilizado para comparação da CC foi o proposto por Freedman e colaboradores (1999).

Os dados coletados por meio da aplicação do questionário foram tabulados e analisados com apoio do software Statistical Package for the Social Sciences (SPSS) a um nível de significância de 5\% (p<0,05) por análise estatística descritiva, e o cálculo das distribuições de frequências, médias e percentuais.

Este estudo foi aprovado pelo Comitê de Ética em Pesquisas - COEP da Universidade Federal de Minas Gerais UFMG sob parecer de $\mathrm{n}^{\circ}$ 16227213.2.0000.5149.

\section{Resultados}

A população do estudo tem faixa etária média de 38,3 com desvio padrão de 8,86 anos, $62,1 \%$ é do sexo feminino ( $n=54)$, e 54,0\% se identifica como parda. Quanto a família, 84,0\% dos entrevistados têm filhos, e 46,0\% são casados.

Em relação a escolaridade, $48,0 \%(n=42)$ dos entrevistados concluíram o ensino médio. Considerando a renda familiar, $63,0 \%(\mathrm{n}=55)$ apresentaram renda de até dois salários-mínimos e meio, e a maioria possui casa própria $(55,0 \%)$.

Entre os trabalhadores entrevistados dos restaurantes, $10,3 \%(n=9)$ apresentaram quadro atual de depressão, e 4,6\% $(\mathrm{n}=4)$ de transtorno de ansiedade. Todos os trabalhadores $(\mathrm{n}=4)$ com diagnóstico de transtorno de ansiedade, também relataram ter depressão. Além disso, 23,0\% $(\mathrm{n}=20)$ dos trabalhadores fazem ou fizeram acompanhamento psicológico, e 8,0\% $(\mathrm{n}=7)$ fizeram ou fazem algum tipo de acompanhamento psiquiátrico. $\mathrm{O}$ uso de antidepressivo foi observado em 12,6\% (n=11) dos trabalhadores entrevistados, e o uso de calmantes, em outros onze $(12,6 \%, \mathrm{n}=11)$ (Tabela 1, Quadro 1). 
Tabela 1 - Características gerais sobre a saúde de trabalhadores de restaurantes universitários. Belo Horizonte/MG, 2017.

\begin{tabular}{|c|c|}
\hline Variáveis & Frequência (\%) \\
\hline Idade (anos, dp) & $33 \pm 8,86$ \\
\hline Homens & $33(37,9)$ \\
\hline Mulheres & $54(62,1)$ \\
\hline \multicolumn{2}{|l|}{ IMC } \\
\hline Eutrofia & $35(42,2)$ \\
\hline Sobrepeso & $29(34,9)$ \\
\hline Obesidade & $19(22,9)$ \\
\hline \multicolumn{2}{|c|}{ Doença ocupacional } \\
\hline Sim & $7(8,1)$ \\
\hline Não & $80(91,9)$ \\
\hline \multicolumn{2}{|c|}{ Acidente de trabalho } \\
\hline Sim & $23(26,4)$ \\
\hline Não & $64(73,6)$ \\
\hline \multicolumn{2}{|l|}{ Hipertensão } \\
\hline Sim & $10(11,5)$ \\
\hline Não & $77(88,5)$ \\
\hline \multicolumn{2}{|l|}{ Diabetes } \\
\hline Sim & $4(4,6)$ \\
\hline Não & $83(95,4)$ \\
\hline \multicolumn{2}{|l|}{ Asma } \\
\hline Sim & $3(3,4)$ \\
\hline Não & $84(96,6)$ \\
\hline \multicolumn{2}{|l|}{ Depressão } \\
\hline Sim & $9(10,3)$ \\
\hline Não & $78(89,7)$ \\
\hline \multicolumn{2}{|c|}{ Transtorno de ansiedade } \\
\hline Sim & $4(4,6)$ \\
\hline Não & $83(95,4)$ \\
\hline \multicolumn{2}{|c|}{ Uso de antidepressivo } \\
\hline $\begin{array}{l}\text { Sim } \\
\text { Não }\end{array}$ & $11(12,6)$ \\
\hline \multicolumn{2}{|l|}{ Uso de calmantes } \\
\hline Sim & $11(12,6)$ \\
\hline Não & $76(87,4)$ \\
\hline \multicolumn{2}{|c|}{ Acompanhamento psicológico n } \\
\hline Sim & $20(23,0)$ \\
\hline Não & $67(77)$ \\
\hline \multicolumn{2}{|c|}{ Acompanhamento Psiquiátrico n } \\
\hline Sim & $7(8,0)$ \\
\hline Não & $80(92,0)$ \\
\hline
\end{tabular}


Além disso, a Tabela 1 descreve variáveis que podem ter impacto na saúde mental do trabalhador como a presença de Doenças Crônicas Não Transmissíveis (hipertensão:11,5\% e diabetes:4,6\%). Outro fator importante na Tabela 1 é o relato de que $26,4 \%$ dos trabalhadores dos restaurantes já tiveram acidentes de trabalho.

$\mathrm{Na}$ avaliação dos dados antropométricos, a média de Índice de Massa Corporal (IMC) foi de $26,8 \mathrm{~kg} / \mathrm{m}^{2}$, sendo 40,0\% $(n=35)$ dos trabalhadores classificados como eutróficos, 33,0\% ( $n=29)$ como sobrepeso, e 27,0\% ( $n=23)$ com algum grau de obesidade. A média da circunferência de cintura (CC) das mulheres foi de $87,1 \mathrm{~cm}$, e dos homens de $87,9 \mathrm{~cm}$ (Tabela 1).

Considerando a função, observou-se que 75,9\% ( $\mathrm{n}=66$ ) são auxiliares de cozinha, 13,8\% (n=12) cozinheiros, três (3) almoxarifes, dois (2) auxiliares de serviços gerais, um (1) auxiliar de almoxarife, um (1) técnico em nutrição e um (1) nutricionista. A maioria tem jornada de trabalho de oito horas diárias, correspondendo a 56,3\% ( $\mathrm{n}=49)$ dos trabalhadores. Quanto ao tempo de trabalho no local, 27,6\% $(n=24)$ trabalham há mais de cinco anos, $21,8 \%(n=19)$ está neste trabalho entre um e dois anos, 19,5\% ( $\mathrm{n}=17)$ há menos de um ano, 13,8\% $(\mathrm{n}=12)$ de dois a três anos, e 9,2\% $(\mathrm{n}=8)$ de três a cinco anos (Tabela 2).

Tabela 2 - Características ocupacionais dos trabalhadores de restaurantes. Belo Horizonte/MG, 2017.

\begin{tabular}{lc}
\hline Variáveis & Frequência n (\%) \\
\hline Função & $2(2,3)$ \\
Auxiliar Serviços Gerais & $66(75,9)$ \\
Auxiliar de Cozinheiro & $1(1,1)$ \\
Auxiliar de Almoxarifado & $12(13,8)$ \\
Cozinheiro & $3(3,4)$ \\
Almoxarife & $2(2,3)$ \\
Técnico em Nutrição & $1(1,1)$ \\
Nutricionista & $49(56,3)$ \\
\hline Jornada de trabalho & $38(43,7)$ \\
8 horas & \\
$12 / 36$ horas & $49(56,3)$ \\
\hline Cursos de capacitação & $38(43,7)$ \\
Sim & Não
\end{tabular}

Fonte: Autores (2017).

A Tabela 2 descreve que $56,3 \%$ dos trabalhadores dos restaurantes já realizaram algum tipo de curso de capacitação na área de manipulação de alimentos.

Entre os trabalhadores com a presença dos Transtornos Mentais Comuns ( $n=9)$, a escolaridade mínima foi de nove anos de estudo, a faixa etária de 26 a 47 anos, e todos são do sexo feminino. Além disso, 66,6\% dos trabalhadores com depressão são auxiliares de cozinha, e 75,0\% daqueles com transtorno de ansiedade também têm essa mesma função.

Quanto ao acompanhamento psicológico, 100\% dos trabalhadores com transtorno de ansiedade e 66,7\% dos trabalhadores com depressão realizam esse acompanhamento. E apenas metade dos trabalhadores diagnosticados com ansiedade (50,0\%), e 44,4\% daqueles com depressão fazem esse tipo acompanhamento psiquiátrico.

O Quadro 1 demostra a associação significativa ou não entre Transtornos Mentais Comuns (TMC) e as variáveis gênero, função, jornada de trabalho, IMC, e realização de acompanhamento psicológico e psiquiátrico pelos trabalhadores de 
restaurantes universitários de Belo Horizonte-MG. De acordo com os resultados obtidos neste estudo, não houve uma associação significativa entre TMC função, jornada de trabalho e IMC. A associação significativa observada foi quanto a gênero e acompanhamento psicológico e psiquiátrico $(\mathrm{p}<0,05)$.

Quadro 1 - Fatores associados aos Transtornos Mentais Comuns (TMC) em trabalhadores de restaurantes universitários de Belo Horizonte/MG, 2017.

\begin{tabular}{|c|c|c|}
\hline \multirow[t]{2}{*}{ Variáveis } & TMC & \multirow{2}{*}{ Valor de $p$} \\
\hline & Frequência n $(\%)$ & \\
\hline \multicolumn{3}{|l|}{ Gênero } \\
\hline Mulher & $9(10,3)$ & \multirow[t]{2}{*}{$0,0132 * *$} \\
\hline Homem & $78(89,7)$ & \\
\hline \multicolumn{3}{|l|}{ Função } \\
\hline Auxiliar Serviços Gerais & $0(0)$ & \multirow{7}{*}{0,3920} \\
\hline Auxiliar de Cozinheiro & $6(6,9)$ & \\
\hline Auxiliar de Almoxarifado & $0(0)$ & \\
\hline Cozinheiro & $2(2,3)$ & \\
\hline Almoxarife & $0(0)$ & \\
\hline Técnico em Nutrição & $1(1,14)$ & \\
\hline Nutricionista & $1(1,14)$ & \\
\hline \multicolumn{3}{|l|}{ Jornada de trabalho } \\
\hline 8 horas & $7(8,0)$ & \multirow{2}{*}{0,1302} \\
\hline $12 / 36$ horas & $2(2,3)$ & \\
\hline \multicolumn{3}{|l|}{ Acompanhamento Psicológico } \\
\hline Sim & $6(6,9)$ & \multirow{2}{*}{$0,0006^{* *}$} \\
\hline Não & $3(3,4)$ & \\
\hline \multicolumn{3}{|c|}{ Acompanhamento Psiquiátrico } \\
\hline Sim & $4(4,6)$ & \multirow{2}{*}{$0,0001 * *$} \\
\hline Não & $5(5,7)$ & \\
\hline \multicolumn{3}{|l|}{ IMC* } \\
\hline Baixo peso & $0(0)$ & \multirow{4}{*}{0,2090} \\
\hline Eutrofia & $3(3,4)$ & \\
\hline Sobrepeso & $2(2,3)$ & \\
\hline Obesidade & $4(4,6)$ & \\
\hline
\end{tabular}

*IMC = Índice de Massa Corporal; **Valor de $p$ significativo no teste qui-quadrado ( $\mathrm{p}<0,05)$. Fonte: Autores $(2017)$.

Desta forma, de acordo com os resultados do Quadro 1 existe uma relação significativa entre gênero, acompanhamento psicológico e psiquiátrico e a presença de TMC nos trabalhadores dos restaurantes universitários. 


\section{Discussão}

Neste estudo observou-se uma prevalência de TMC de 10,3\% entre os trabalhadores dos restaurantes, valor semelhante à prevalência de depressão para o Brasil (10\%) (Santana et al., 2016). Em estudos com outros grupos de trabalhadores foram encontrados valores semelhantes de Transtornos Mentais Comuns (TMC) aos obtidos nesta pesquisa. Entre eles, o estudo de Uchoa e colaboradores (2010) sobre TMC com motoristas de caminhões cuja prevalência de TMC foi de $6,1 \%$.

Neste estudo, de acordo com os dados obtidos houve associação significativa $(\mathrm{p}<0,05)$ entre gênero e a presença de Transtornos Mentais Comuns (TMC) entre os trabalhadores de restaurantes.

Em se tratando da categoria gênero, diversos estudos realizados sobre TMC, identificam a prevalência desta doença mental entre as mulheres (Carlotto, Barcinski \& Fonseca, 2015).

A relação entre o gênero feminino e TMC também foi observada por Cunha e colaboradores (2012) em que descrevem a prevalência de depressão em população de baixa renda de Porto Alegre, Rio Grande do Sul, e por Wang e colaboradores (2008) em estudo sobre relação entre transtornos mentais e sexo, no Canadá. Fleck e colaboradores (2003) constataram que a depressão incide de duas a três vezes mais em mulheres do que em homens, mesmo em diferentes países.

$\mathrm{Na}$ literatura é possível perceber a prevalência de transtornos mentais em mulheres, entretanto, não é possível relacionar com outros fatores que justifiquem essa prevalência. Essa falta de fatores que justifique as mulheres serem mais afetadas pelas doenças mentais é comumente relatada por diferentes autores, sendo necessário mais estudos sobre esta temática (Ludermir, 2008; Kapper, 2014; Borges, Hegadoren \& Miasso, 2015).

Diferentemente do encontrado no estudo de Cunha e colaboradores (2012) em que a prevalência de depressão foi maior em indivíduos com menos de quatro anos de escolaridade, nesta pesquisa os trabalhadores com depressão têm no mínimo nove anos de estudo. Já Parreira e colaboradores (2017) observaram que para cada ano a mais de estudo diminuem em $12 \%$ as chances de a mulher ter TMC, em estudo realizado com mulheres de uma área rural.

A obesidade é um problema de saúde multifatorial e, quando associado a comorbidades, acarreta maiores complicações tanto nos aspectos terapêuticos como evolutivos. Independente de sua gravidade é primordial a observância dos seus fatores etiológicos, desencadeantes, predisponentes e perpetuadores. Encontram-se entre os seus fatores de risco, ainda, a associação com distúrbios emocionais, como a ansiedade e a depressão, os quais muitas vezes aumentam a necessidade da ingestão de alimentos calóricos ou desregulam o ritmo alimentar e metabólico, trazendo complicações ao tratamento e comprometendo o prognóstico do quadro clínico (Casselli et al., 2021).

Há evidências de que a obesidade e os transtornos mentais, especialmente depressão e ansiedade estão associadas de maneira bilateral, ou seja, a obesidade pode aumentar a incidência de depressão e ansiedade, e os transtornos mentais favorecem o desenvolvimento da obesidade (Simon et al., 2006). Além de possível ganho de peso e alterações metabólicas devido ao uso de medicações psiquiátricas que interferem na fome e saciedade (Berkowitz \& Fabricatore, 2011). Nos transtornos mentais e na obesidade, o estresse, hábitos de vida, experiências traumáticas, humor, estado emocional e relacionamentos interagem com processos inflamatórios e cargas genéticas, ocasionando à desregulação de sistemas neuroimunoendócrinos (Melca \& Fortes, 2014).

A obesidade pode contribuir para a baixa autoestima, insatisfação corporal e alteração da autoimagem, sendo considerados fatores de risco para o desenvolvimento da depressão (de Wit et al., 2010).

Predominantemente, a depressão diminui a motivação e causa sensação de falta de energia, além de influenciar nos hábitos alimentares e físicos, podendo levar à uma diminuição na preocupação com a saúde. Quem possui depressão realiza menos ou nenhuma atividade física, favorecendo o desequilíbrio calórico, ou seja, a ingestão calórica é geralmente maior que o gasto calórico (Verdolin et al., 2012). 
De acordo com Verdolin e colaboradores (2012), o transtorno de ansiedade é mais prevalente em pacientes obesos do que com sobrepeso, o que também foi observado neste estudo, onde $75 \%$ dos trabalhadores com transtorno de ansiedade possuem algum grau de obesidade, e nenhum possui sobrepeso. Neste estudo, apesar de não ter sido encontrada associação entre estado nutricional e TMC, observou-se que em 6,9\% da população estudada que possui TMC encontra-se com algum grau de obesidade.

Para a função exercida, nota-se que a maior parte dos indivíduos com TMC nos restaurantes são auxiliares de cozinha, cargo que sofre grande pressão pela alta carga de trabalho advinda das funções exercidas por estes trabalhadores e curtos prazos para realização das atividades, além de possuir pouca autonomia na tomada de decisões. Outro trabalhador que apresentou TMC foi a técnica em nutrição. Wang e colaboradores (2008) mostraram que trabalhadores que têm pouco controle na tomada de decisões e alta demanda de serviço apresentam maior associação com os transtornos mentais.

Em um estudo realizado sobre a avaliação da qualidade de vida com manipuladores de alimentos em restaurantes verificou-se que os domínios mais comprometidos foram dor e vitalidade, enquanto a capacidade funcional obteve a melhor pontuação. Os adultos mais jovens apresentaram melhor vitalidade e saúde mental quando comparados aos mais velhos, que obtiveram pior capacidade funcional. Os cozinheiros tiveram melhor resultado no aspecto físico. Os homens com menor escolaridade alcançaram melhor estado geral de saúde e os com maior renda melhor aspecto social e emocional. A qualidade de vida dos manipuladores foi considerada satisfatória e apresenta variações relacionadas à faixa etária, escolaridade e renda (Nalle et al., 2018).

A grande maioria dos trabalhadores com TMC atualmente realizam acompanhamento psicológico ou psiquiátrico. Neste estudo, observou-se associação significativa entre o acompanhamento psicológico e ou psiquiátrico e TMC (depressão e ou transtorno de ansiedade) nos trabalhadores de restaurantes.

Cada realidade de trabalho tem suas peculiaridades, principalmente relacionadas aos riscos à saúde, ao grau do estresse gerado pela atividade, ao tempo de exposição dos trabalhadores às altas cargas físicas, cognitivas e psíquicas e a sua contribuição para a incidência de doenças ocupacionais (Benetti et al., 2014; Ulhôa et al., 2010).

Alguns estudos já demonstram a relação dos TMC e doenças ocupacionais em trabalhadores de restaurantes. Haukka e colaboradores (2011) investigaram a relação entre o estresse mental e fatores psicossociais no trabalho (controle do trabalho, discernimento de habilidade, suporte gerencial, relações interpessoais no trabalho e pressão temporal) e a predição de dores musculoesquelética entre mulheres que trabalham em cozinhas. Haruyama e colaboradores (2014) encontraram correlação entre a ocorrência de queimaduras e cortes relacionados ao trabalho na cozinha com os níveis de estresse no trabalho entre cozinheiros industriais.

Os fatores de risco ergonômicos e psicossociais relacionados ao trabalho, ou seja, o ambiente físico, a carga de trabalho elevada, e as dificuldades nas relações socioprofissionais podem ser consideradas variáveis que interferem na saúde mental e física do trabalhador, de acordo com o estudo realizado por Sticca, Mandarini \& Silva (2019).

Pesquisadores têm buscado rastrear as atividades realizadas pelos trabalhadores em restaurantes, com o intuito de elaborar estratégias que minimizem a propagação de doenças e auxiliem na prevenção da saúde e segurança dos seus trabalhadores (Epifânio, Rique, \& Nascimento, 2020).

Consideram-se como limitações do estudo a baixa validade externa, por ter sido realizado em uma amostra de conveniência. Por outro lado, têm-se como pontos fortes os cuidados metodológicos de coleta de dados para que os resultados expressassem de fato a realidade vivenciada pelos trabalhadores dos restaurantes.

Os dados deste estudo contribuem para a avaliação da saúde mental no setor de Alimentação Coletiva e demonstram a existência de Transtornos Mentais Comuns (TMC) em trabalhadores de restaurantes universitários. Ressalta-se, o papel do gestor do restaurante na condução das atividades a frente da equipe, bem como em proporcionar condições ambientais 
adequadas para execução das atividades laborais a fim de favorecer conforto e saúde ao trabalhador e proporcionar a sua produtividade.

\section{Conclusão}

$\mathrm{O}$ trabalho nos restaurantes constitui-se em um processo complexo com interações técnicas e sociais. Assim, faz-se necessário ter em conta a satisfação dos profissionais quanto à organização e processos de produção das refeições, além da implementação de programas que visem à melhoria da atenção à saúde.

Os resultados deste estudo indicaram que a prevalência de TMC entre os trabalhadores de restaurantes é uma realidade e que necessita de intervenção para promover melhor qualidade de vida a esses indivíduos. Todavia, ainda, são necessários mais estudos baseados em outros aspectos que poderão ser explorados para contribuir na melhoria da qualidade de vida dos trabalhadores dos restaurantes.

Assim, sugere-se que outros estudos possam ser realizados com diferentes tipos de restaurantes como comerciais e hospitalares, de forma a proporcionar uma visão mais ampliada da saúde mental desta classe de trabalhadores.

\section{Referências}

Abreu, E. S., Spinelli, M. G. N., \& Pinto, M. A. S. (2019) Gestão de Unidades de Alimentação e Nutrição: um modo de fazer. (2a ed.), Editora Metha.

Barreto, A. C., \& Branco, A. B. (2000) Influência da atividade física sistematizada no estresse e na fadiga dos trabalhadores do restaurante universitário da Universidade de Brasília. Revista Brasileira de Atividade Física e Saúde, 5(2), 23-29. https://doi.org/10.12820/rbafs.v.5n2p23-29

Bárbaro, A. M., Robazzi, M. L. D. C. C., Pedrão, L. J., Cyrillo, R. M. Z., \& Suazo, S. V. V. (2009) Transtornos mentais relacionados ao trabalho: revisão de literatura. SMAD, Revista Eletrônica Saúde Mental, Álcool e Drogas, 2009; 5(2):1-16. https://doi.org/10.11606/issn.1806-6976.v5i2p1-18

Berkowitz, R. I., \& Fabricatore, A. N. (2011) Obesity, Psychiatric Status, and Psychiatric Medications. Psychiatric Clinics of North America Journal, 34(4), 747-64. https://doi.org/10.1016/j.psc.2011.08.007

Benetti, S. P. C., Schwartz, C., Soares, G. R., Macarena, \& F., Patussi, M. M. (2014) Psychosocial adolescent psychosocial adjustment in Brazil - perception of parenting style, stressful events and violence. International Journal of Psychological Research, 7(1), 40-48. https://doi.org/10.21500/20112084.666

Borges, T. L., Hegadoren, K. M., \& Miasso, A. I. (2015). Transtornos mentais comuns e uso de psicofármacos em mulheres atendidas em unidades básicas de saúde em um centro urbano brasileiro. Revista Panamericana de Salud Publica, 38(3), 195-201. https://www.scielosp.org/article/rpsp/2015.v38n3/195-201/

Carlotto, M. S., Barcinski, M., \& Fonseca, R. (2015) Transtornos mentais comuns e associação com variáveis sociodemográficas e estressores ocupacionais: uma análise de gênero. Estudos e Pesquisas em Psicologia, 15(3), 1006-1026. publicacoes.uerj.br/index.php/revispsi/article/viewFile/19424/14101

Casselli, D. D. N., Silva, E. S. M., Figueira, G. M., Demarchi, M. E., \& Souza, J. C. (2021) Comorbidade entre depressão, ansiedade e obesidade e complicações no tratamento. Research, Society and Development, 10(1), e16210111489. http://dx.doi.org/10.33448/rsd-v10i1.11489

Colares, L. G. T., Freitas, \& C. M. F. (2007) Processo de trabalho e saúde de trabalhadores de uma unidade de alimentação e nutrição: entre a prescrição e o real do trabalho. Cadernos de Saúde Pública, 23(12), 3011-20. https://doi.org/10.1590/S0102-311X2007001200022

Cruz Neto, J., Feitosa, E. M. S., Sidrim, A. C., Morais, J. M. T. S., \& Oliveira, C. C. (2020) Sintomas associados a transtornos mentais comuns no contexto universitário. Research, Society and Development, 9(11), e47091110000. http://dx.doi.org/10.33448/rsd-v9i11.10000

Cunha, R. V., Bastos, G. A. N., \& Duca, G. F. D. (2012) Prevalência de depressão de fatores associados em comunidade de baixa renda de Porto Alegre, Rio Grande do Sul. Revista Brasileira de Epidemiologia, 15, 356-54. https://doi.org/10.1590/S1415-790X2012000200012.

de Wit, L. M, Fokkema, M., Van Straten, A., Lamers, F., Cuijpers, P., \& Penninx, B. W. (2010) Depressive and anxiety disorders and the association with obesity, physical and social activities. Depress Anxiety, 27(11), 1057-65. https://doi.org/10.1002/da.20738

Dourado, M. M. J., \& Lima, T. P. (2011) Ergonomia e sua importância para os trabalhadores de Unidades de Alimentação e Nutrição. Ensaios e Ciências: Ciências Biológicas, Agrárias e da Saúde, Valinhos, 15(4), 183-96. https://revista.pgsskroton.com/index.php/ensaioeciencia/article/view/2870

Epifânio, M. A. B., \& Rique, T. P. (2020) Nascimento, S. M. Saúde e sofrimento do trabalhador atuante em restaurantes universitários: uma breve revisão. Brazilian Journal of Development, 6(11), 93678-93693. https://doi.org/10.34117/bjdv6n11-689

Estevam, E., \& Guimarães, M. (2013) Caracterização do perfil nutricional e dos aspectos ergonômicos relacionados ao trabalho de colaboradores de uma unidade de alimentação e nutrição. Revista Científica Faminas, 9(2), 55-68. https://www.faminasbh.edu.br/upload/downloads/20131227152436_529478.pdf

Fischer, F. M. (2012) Relevância dos fatores psicossociais do trabalho na saúde do trabalhador. Revista de Saúde Pública, 46(3), 401-06. https://doi.org/10.1590/S0034-89102012000300001 
Fleck, M. P. de A., Lafer, B., Sougey, E. B., Del Porto, J. A., Brasil, M. A, \& Juruena, M. F. (2003). Diretrizes da Associação Médica Brasileira para o tratamento da depressão (versão integral). Revista Brasileira de Psiquiatria, 25(2), 114-122. https://doi.org/10.1590/S1516-44462003000200013.

Freedman, D. S., Serdula, M. K., Srinivasan, S.R., \& Berenson, G.S. (1999) Relation of circumference and skinfold thicknesses to lipid and insulin concentrations in children and adolescents: The Bogalusa Heart Study. The American Journal Clinical Nutrition, 69, 308-3017. https://doi.org/10.1093 / ajcn / 69.2 .308

Ganster, D. C., \& Rosen, C. C. (2013) Work stress and employee health: a multidisciplinary review. Journal of Management, $39(5)$, $1085-1122$. HTTPS://DOI.ORG/10.1177/0149206313475815

Goldberg, D. P., \& Huxley, P. (1992) Common mental disorders: a bio-social model. Tavistock/Routledge.

Härmä, M., Kompier, M. A., \& Vahtera, J. (2006) Work-related stress and health-risks, mechanisms and countermeasures. Scandinavian Journal of Work, Environment \& Health, 32(6), 413-19. https://doi.org/10.5271 / sjweh.1047.

Haruyama, Y., Matsuzuki, H., Tomita, S., Muto, T., Haratani, T., Muto, S., \& Ito, A. (2014) Burn and cut injuries related to job stress among kitchen workers in Japan. Industrial Health, 52(2), 113-120. https://doi.org/10.2486 / indhealth.2013-0143

Haukka, E., Leino-Arjas, P., Ojajärvi, A., Takala, E. P., Viikari Juntura, E., \& Riihimäki, H. (2011) Mental stress and psychosocial factors at work in relation to multiple-site musculoskeletal pain: A longitudinal study of kitchen workers. European Journal of Pain, 15(4), 432-438. https://doi.org/10.1016/j.ejpain.2010.09.005

Kaspper, L. S., \& Schermann, L. B. (2014) Prevalência de transtornos mentais comuns e fatores associados em usuárias de um Centro de Referência de Assistência Social de Canoas/RS. Aletheia, 45, 168-176. http://pepsic.bvsalud.org/scielo.php?script=sci_abstract\&pid=S1413$03942014000200013 \& \operatorname{lng}=\mathrm{pt} \& \mathrm{nrm}=\mathrm{iso} \& \operatorname{lng}=\mathrm{en}$

Konzen, I. G. D. N. C., Konzen, M. R., da Silva Neto, J. M., de Freitas Carneiro, A., \& de Brito Almeida, A. G. (2020) Desafios para garantir a aplicação das estratégias preventivas de segurança no trabalho na construção civil. Brazilian Journal of Development, 6(8), 56752- 56776. https://doi.org/10.34117/bjdv6n8-185

Lima, D. P., Sties, S. W., Gonzales, A. I., Bundchen, D. C., Aquino, G. I., Carvalho, T., Neto Schimitt, A., \& Fontes, Y. G. S. (2016) Questionário para avaliação da dor musculoesquelética em praticantes de exercícios (Q-ADOM). Revista Brasileira de Medicina do Esporte, 22(5), 374-380. https://doi.org/10.1590/1517-869220162205161347

Lindström, M. (2005) Psychosocial work conditions, unemployment and self-reported psychological health: a population-based study. Occupational Medicine, 55(7), 568-71. https://doi.org/10.1093 / occmed / kqi122

Lopes, C. S., Faerstein, E., \& Chor, D. (2003) Eventos de vida produtores de estresse e transtornos mentais comuns: resultados do Estudo Pró-Saúde. Cadernos de Saúde Pública, 19(6), 1713-1720. https://doi.org/10.1590/S0102-311X2003000600015.

Lourenço, M. S., \& Menezes, L.F. (2008) Ergonomia e alimentação coletiva: análise das condições de trabalho em uma Unidade de Alimentação e Nutrição. In: Congresso Nacional de Excelência em Gestão. Responsabilidade Socioambiental das Organizações Brasileiras. Niterói, RJ.

Ludermir, A. B. (2008) Desigualdades de classe e gênero e saúde mental nas cidades. Revista de Saúde Coletiva, 18(3), 451-467. https://doi.org/10.1590/S0103-73312008000300005

Melca, I. A., \& Fortes, S. (2014) Obesidade e transtornos mentais: construindo um cuidado efetivo. Revista HUPE, 13(1), 18-25. https://www.epublicacoes.uerj.br/index.php/revistahupe/article/view/9794

Melo, C. C. M., Bernardes, L. F., Morceli, G., Silva, P. G., Pereira, S. S., \& Santos, S. V. M (2021) Nível de evidência dos estudos relacionados à ansiedade, estresse e depressão dos profissionais de enfermagem. Research, Society and Development, 10(1), e2210111295. http://dx.doi.org/10.33448/rsd-v10i1.11295

Nalle, G. S., Vieira, P. M., Sousa, A. A., Pacheco, E. A. C., Aragão, A. S., \& Dutra, F. C. M. S (2018) Qualidade de vida de manipuladores de alimentos em restaurantes industriais. REFACS - Revista Família, Ciclos da Vida e Saúde no Contexto Social (online), 6(Supl. 2), 581-590.

Niedhammer, T., Malard, L., \& Chastang, J. F. (2015) Occupational factors and subsequent major depressive and generalized anxiety disorders in the prospective French national SIP study. BioMed Central Public Health. Paris, France.

Organização Mundial de Saúde (2017) Depression and Other Common Mental Disorders: Global Health Estimates. WHO MDS. Disponível em: <http://apps.who.int/iris/bitstream/handle/10665/254610/WHO-MSD-MER-2017.2eng.pdf;jsessionid=316708BD6B1B93C4CF1AC24F5623D829? sequence $=1>$

Parreira, B. D. M., Goulart, B. F., Haas, V. J., Silva, S. R., Monteiro, J. C. S., \& Gomes-Sponholz, F. A. (2017) Transtorno mental comum e fatores associados: estudo com mulheres de uma área rural. Revista da Escola de Enfermagem USP, 51, e03225. http://dx.doi.org/10.1590/S1980$220 \times 2016033103225$

Pereira A. S., Shitsuka, D. M., Parreira, F. J., \& Shitsuka, R. (2018). Metodologia da pesquisa científica. UFSM. https://repositorio.ufsm.br/bitstream/handle/1/15824/Lic_Computacao_Metodologia-Pesquisa-Cientifica.pdf?sequence=1

Pinto, I. C. M., \& Silva, I.V. (2016) Condições de Trabalho e o Sofrimento Invisível dos Trabalhadores da Saúde. In: Braga IFB, Velasques MCC, Morosini MVC, Batistella RRC, Lima JCF, Chagas DC. O trabalho no mundo contemporâneo: fundamentos e desafios para a saúde. (22a ed.), Editora Fiocruz.

Santana, L. L., Sarquis, L. M. M., Brey, C., Miranda, F. M. D., \& Felli, V. E. A. (2016). Absenteísmo por transtornos mentais em trabalhadores de saúde em um hospital no sul do Brasil. Revista Gaúcha de Enfermagem,37(1). https://doi.org/10.1590/1983-1447.2016.01.53485 
Research, Society and Development, v. 10, n. 3, e21410313212, 2021

(CC BY 4.0) | ISSN 2525-3409 | DOI: http://dx.doi.org/10.33448/rsd-v10i3.13212

Silva, M. P., Bernardo, M. H., \& Souza, H. Á. (2016) Relação entre saúde mental e trabalho: a concepção de sindicalistas e possíveis formas de enfrentamento. Revista Brasileira de Saúde Ocupacional, 41, e-23. https://doi.org/10.1590/2317-6369000003416.

Silva, M. C., \& Borges, L. O. (2015) Condições de trabalho e clima de segurança dos operários da construção de edificações. Revista Psicologia, Organizações e Trabalho, 15(4), 407-418. https://doi:10.17652/rpot/2015.4.626

Simon, G. E., Von Korff, M., Saunders, K., Miglioretti, D. L., Crane, P. K., Van Belle, G., \& Kessler, R. C. (2006) Association between obesity and psychiatric disorders in the US adult population. Archives of General Psychiatry, 63(7), 824-30. https://doi.org/10.1001 / archpsyc.63.7.824

Sticca, M. G., Mandarini, M. G., \& Silva, F. H. M. (2019) Condições de trabalho e saúde de trabalhadores em um restaurante universitário. Estudos e Pesquisas em Psicologia, 13(3), 583-603. http://pepsic.bvsalud.org/pdf/epp/v19n3/v19n3a03.pdf

Sousa, K. A., Freitas, F. F. Q., Castro, A. P., Oliveira, C. D. B., Almeida, A. A. B., \& Sousa, K. A. (2017). Prevalência de sintomas de depressão em idosos assistidos pela estratégia de saúde da família. Revista Mineira de Enfermagem, 21, 1-7. https://doi.org/10.5935/1415- 2762.20170028

Ulhôa, M. A., Marqueze, E. C., Lemos, L. C., Silva, L. G., Silva, A. A., Nehme, P., Fischer, F. M., \& Moreno, C. R. C. (2010) Distúrbios psíquicos menores e condições de trabalho em motoristas de caminhão. Revista de Saúde Pública, 44(6), 1130-36. https://doi.org/10.1590/S0034-89102010000600019

Tenório, L. P., Argolo, V. A., de Sá, H. P., Melo, E. V., \& Costa, E. F. O. (2016) Saúde mental de estudantes de escolas médicas com diferentes modelos de ensino. Revista Brasileira de Educação Médica, 40(4), 547-582. https://doi.org/10.1590/1981-52712015v40n4e00192015

Verdolin, L. D., Borner, A. R. S., Guedes Jr, R. O., da Silva, T. F. C., Belmonte, \& T. S. A. (2012) Comparação entre a prevalência de transtornos mentais em pacientes obesos e com sobrepeso. Scientia Médica, 22(1), 25-31. https://revistaseletronicas.pucrs.br/ojs/index.php/scientiamedica/article/view/9918/7531

Wang, J. L., Lesage, A., Schmitz, A., \& Drapeau, A. The relationship between work stress and mental disorders in men and women: findings from a population-based study. Journal of Epidemiology Community Health, 62(1), 42-47. https://doi.org/10.1136 / jech.2006.050591

World Health Organization - WHO (2017). Depression and Other Common Mental Disorders $\quad-\quad$ Global Health Estimates. http://apps.who.int/iris/bitstream/10665/254610/1/WHO-MSD-MER-2017.2-eng.pdf?ua=1.

World Health Organization - WHO (2000) Obesity: preventing and managing the global epidemic. Geneva: WHO https://apps.who.int/iris/handle/10665/42330

World Health Organization - WHO (2020). Saúde mental: fortalecendo nossa resposta. https://www.who.int/news-room/fact-sheets/detail/mentalhealthstrengthening-our-response 